\title{
ST Değişiminin Wigner-Ville Dağılım Esaslı Erken Tespiti
}

\section{İlknur KAYIKÇIOĞLU*1,3, Güzin ULUTAŞ ${ }^{1}$, Fulya AKDENiZ² ${ }^{2}$, Temel KAYIKÇIOĞLU ${ }^{2}$}

\author{
${ }^{1}$ Karadeniz Teknik Üniversitesi, Mühendislik Fakültesi, Bilgisayar Mühendisliği Bölümü, 61080, Trabzon \\ ${ }^{2}$ Karadeniz Teknik Üniversitesi, Mühendislik Fakültesi, Elektrik-Elektronik Mühendisliği Bölümü, 61080, \\ Trabzon \\ ${ }^{3}$ Zonguldak Üniversitesi, Mühendislik Fakültesi, Bilgisayar Mühendisliği Bölümü, 67980, Trabzon
}

(Alınış / Received: 30.12.2016, Kabul / Accepted: 11.09.2017, Online Yayınlanma / Published Online: 24.10.2017)

\section{Anahtar Kelimeler}

Elektrokardiyagram(EKG),

ST değișimi,

Myokard enfarktüs,

Wigner-Ville dağılımı, Teletıp

\begin{abstract}
Özet: EKG işaretinde ST değişiminin erken tespit edilmesi myokard enfarktüs önlenmesi açısından oldukça önemlidir. Bu çalışmada ST değişimin erken tespit etmek amacıyla Wigner-Ville dağlımına dayanan bir algoritma geliştirilmiştir. Algoritma MIT-BIH Aritmi ve European ST-T veritabanlarından üretilen büyük bir veride test edilmiştir. MIT-BIH veritabanından V1, V2, V4, V5 derivasyonlarında sağlıklı veya aritmi içeren 111688 R-R aralığı ve European ST-T veritabanından V1, V2, V3, V4, V5 derivasyonlarında 111688 tane ST değișimi olan R-R aralıkları seçilmiştir. Sınıflandırmada performans sonuçları doğruluk, duyarlılık, özgüllük ve pozitif öngörü, sırasıyla \%98,78, \%98,55, \%99,0 ve \%99,01 olarak bulunmuş olup bu değerler literatürdeki çalışmalara ait değerlerin üstündedir. Ayrıca önerilen algoritmanın hızı tele-tıp sistemleri için oldukça uygundur.
\end{abstract}

\section{Early Detection of ST Change Based on Wigner-Ville Distrubition}

\section{Keywords}

Electrocardiogram (ECG), ST change,

Myocardial infarction, Wigner-Ville distribution, Telemedicine

\begin{abstract}
Early detection of ST change in ECG signals is very important for the prevention of myocardial infarction. In this study, a Wigner-Ville distribution based algorithm was developed for early detection of ST change. The algorithm was tested on big data which was were generated from the MIT-BIH database and the European ST-T database. From the MIT-BIH database, 111688 R-R intervals containing healthy or arrhythmias in V1, V2, V4, V5 leads and R-R intervals with 111688 ST segment changes in V1, V2, V3, V4, V5 derivations from the European ST-T database were selected. Performance results in classification are obtained as for accuracy, sensitivity, specificity and positive predictive, respectively $98,78 \%$, $98,55 \%, 99,01 \%$ and $99,01 \%$, these values are over of those of studies in literature. The speed of the algorthim is also suitable for telemedicine systems.
\end{abstract}

\section{Giriş}

Tıp ve bilim teknik alanındaki ilerlemelere bağlı olarak ortalama insan yaşam süresi artmakta ve buna bağlı olarak dünyadaki yaşlı nüfus oranı gün geçtikçe yükselmektedir. $\mathrm{Bu}$ durum başta kronik kalp rahatsızlıkları olmak üzere birçok hastalığı beraberinde getirmektedir [1]. Dünya sağlık örgütü (WHO)'nün yaptığı araştırmalara göre her yıl 17,5 milyon insan kalp ve damar hastalıkları yüzünden hayatını kaybetmektedir. Bu da dünyadaki ölümlerin yaklașık \%31 ine denk gelmektedir. Ayrıca kalp hastalıklarına bağlı meydana gelen ölümlerin \%80'ini kalp krizi ve felç oluşturmaktadır [2].

Myokard enfarktüs (MI) kalp atardamarlarının tamamının tıkanması ya da kalp kası hücrelerinde kan akışının azalması sonucu meydana gelir [3]. MI dünyadaki kalp rahatsızlıklarına bağlı ölüm ve sakatlıklarının en büyük sebeplerinden biridir [4].

MI Holter esaslı EKG sinyallerinden, biyokimyasal belirleyicilerden ve Ekokardiyografi görüntüleme ile teşhis edilebilir [5]. MI belirtileri EKG sinyalinin ST kısmındaki değișimlerden tespit edilebilir [6]. Suskun iskemi (silent ischemia) uzun dönemli olup yavaş gelişmektedir [7]. ST kısmındaki ciddi değişimler kalp krizinden saatlerce önce olușmakta ve kalp krizi süresince devam etmektedir [3]. Yukarıda izah edilen teşhis yöntemlerinde; bir zaman gecikmesi söz konusu olduğu için tedavi etkinliği düşük olmaktadır. Bundan dolayı EKG sinyali gerçek zamanlı izlenerek ST kısmındaki değişimlerin erken tespiti edilmesi tedavinin etkinliği için oldukça önemlidir. Bilgi ve 
teknoloji alanındaki gelişmeler hastaların Tele-Tıp sistemleri ile daha az bir maliyet ve yüksek yaşam kalitesi ile takip edilmesine imkan tanımaya başlamıștır. Bu sistemler hastada oluşabilecek sağlık problemlerini önceden bulur ve müdahalenin daha kısa sürede yapılmasını ve gerekli önlemlerin alınmasına olanak sağlar [8].

$\mathrm{Bu}$ çalışmada ST değişimini erken tespit edebilecek bir sınıflandırma algoritmasının geliştirilmesi hedeflenmiştir. Algoritma Wigner-Ville esaslı öznitelikleri kullanmakta olup; performans analizi birçok sınıflandırma tekniklerinde yapılmış ve literatürde bulunan yöntemlerle karşılaştırılmıştır. $\mathrm{Bu}$ konu ile ilgili çalışmalar bir sonraki kısımda özetlenmiştir. Üçüncü bölümde geliştirilen algoritma ayrıntılı bir şekilde izah edilmiştir. Algoritmanın performans sonuçları ve diğer yöntemlerle karşılaştırılması sırasıyla Bölümler 4 ve 5 'de ve son olarak da Teşekkür ve Referans bölümleri verilmiştir.

$\mathrm{Bu}$ alanda birçok araştırmacı farklı öznitelikler, sınıflandırma yöntemleri ve farklı veritabanları kullanarak ST kısmındaki değişimleri tespit etmeye çalışmışlardır. Bu çalışmalarda öznitelikler, Dalgacık Dönüşümü [9-10], Karhunen-Loeve Dönüşümü (KLT) [11-12], Genetik Algoritması [13], Saklı Markov Modeli (HMM) [14], Vektörkardiyografi (kalp yöney eğrisi çizim yöntemi) ST-T Metodu [15] ve Saklı Markov Modeli (HMM) ve Gauss Karışım Modeli (GMM)[16] kullanılarak elde edilmiştir. Çalışmamızda ise Wigner-Ville esaslı öznitelikler geliştirilmiştir.

Xu vd. [3], şekilsel sinıflandırmayla ST bölümünü tespit etmişlerdir. Toplamda 17,314 EKG atımı European ST-T veritabanından alınmıştır. Eğitim ve test doğrulukları \%91,8 ve \%90,1 olarak verilmiştir. Liu ve diğerleri [6] çalışmalarında EKG işaretine 20.dereceden polinom uydurmuşlardır. Karar Destek Makineleri, Rastgele Ağaçlar, Karar Ağaçları ve Naif Bayes sinıflandırma algoritmaları kullanarak MI tespit etme doğruluğu en yüksek \%94,4 bulunmuştur. Çalışmanın EKG sinyalleri Physikalisch-Technische Bundesanstalt veritabanından (PTBdb) alınmış olup standart 12 derivasyon kullanılmıștır. Çalıșmada 148 ST değimli ve 52 sağlıklı veri seçilmiştir. Exarchos vd. [17], EKG verilerini European Society of Cardiology ST-T veritabanından seçmişlerdir. Veritabanından e0104 numaralı verinin 2 saati yani tamamı e0103, e0105, e0108, e0113, e0114, e0147, e0159,e0162 ve e0206 numaraları verilerinin 1 saati seçilmiştir. $\mathrm{Bu}$ seçim sonucunda 86384 kalp atımı normal veya iskemi olarak etiketlenmiștir. Duyarlılı \%87 ve özgüllük \%93 değerlerini tespit etmişlerdir. Dranca vd. [18], çalışmalarında Karar Destek Ağaçları kullanarak gerçek zamanlı ST değişinin tespitini sağlamıștır. Veritabanı olarak Long Term ST veritabanı seçilmiştir. Duyarlılı ve pozitif öngörü değeri sırasıyla \%83,33 \%77,31 olarak tespit etmişlerdir. Tang vd. [19], Long Term ST-T veritabanını kullanmışlardır. Çalışmada Saklı Markov Modeli (HMM) kullanılarak öznitelik çıkartımı gerçekleștirilmiştir. Duyarlılık ve pozitif öngörü değeri sırasıyla \%89 ve \%85 verilmiştir. Al-Fahaum vd. [20], rastgele 14 tane hastalıklı EKG sinyalini European ST-T veritabanından ve 24 tane rastgele normal EKG sinyallerini MIT-BIH Aritmi veritabanından seçmişlerdir. Yüksek Frekans Analizi teknikleri uygulanarak ortalama duyarlılık ve pozitif öngörü değeri sırasıyla \% 100 ve \%93,33 bulmuşlardır. Papaloukas vd. [21], Kural Tabanlı Atım sinıflandırmasıyla hastalıklı bölüm tespiti sağlamışlardır. Bu yöntem ESC ST-T veritabanında test edilerek duyarlılı ve pozitif öngörü değeri sırasıyla \%93,8 ve \%78,5 olarak verilmiştir. Kumar vd. [22], ST segmentindeki sapmalar sonucu oluşan İzoelektrik Enerji fonksiyonu kullanarak MI tespitini sağlamışlardır. European ST-T veritabanından seçtikleri e0103, e0104, e0105, e0108, e0113, e0114,e0147, e0159, e0162,e0206 numaralı verilerden toplamda 43,876 ST bölümü almışlardır. Ortalama duyarlılı \%98,12 özgüllükse \%98,16 olarak belirlenmiştir. Yukarıda verilen çalıșmalar ve çalışmamızın sonuçları karşılaştırma amacıyla Tablo 4'de verilmiştir.

$\mathrm{Bu}$ çalışmada MIT-BIH Aritmi ve European ST-T veritabanları birlikte kullanılmıştır. Toplamda 223376 tane hastalıkl, sağlıklı ve ST değișimi içeren R-R aralıkları seçilip literatürdeki çalışmalara göre daha büyük bir veri tabanı oluşturulmuştur.

\section{Materyal ve Metot}

$\mathrm{Bu}$ çalışmada EKG sinyalindeki herhangi bir R-R aralığındaki ST kısmında bir değişim olup olmadığını tespit edebilecek bir algoritmanın gerçekleştirilmesi amaçlanmıştır. Algoritmada giriş olarak sadece R-R aralığı alınarak öznitelik çıkartımı işlemi sağlanmıştır. Ayrıca algoritma hiçbir filtrelemeye ihtiyaç duymamaktadır. Algoritmanın büyük bir veritabanında eğitimi ve testi yapılmıştır. Yöntemde kullanılan Veritabanı, Önişleme, Öznitelikler ve Sınıflandırma aşamaları sırasıyla aşağıda açıklanmıştır.

\subsection{Veritabanı}

Veriler MIT-BIH aritmi veritabanı ve European ST-T veritabanından alınmıștır [23]. MIT-BIH veritabanından V1, V2, V4, V5 derivasyonları seçilmiştir. $\mathrm{Bu}$ veritabanında 13 farklı aritmi tipi kullanılmıştır. Bunlar sol dal bloğu (LBBB), sağ dal bloğu (RBBB), erken kulakçık atımları (AP), anormal erken kulakçık atımları (aAP), süpraventriküler ektopik atım veya erken ektopik atım, ventriküler erken karıncık atımı (PVC), normal ve karıncık atım füzyonu (fVN), nodal (junctional) kaçak atım (NE),ventriküler kaçak atım (VE), yapay vuru, yapay ve normal vuru füzyonu (fPN), ventriküler flutter dalga, nodal (junctional) erken atım (NP)'dır.

Toplamda sağlıklı veya aritmi içeren 111688 R-R aralığı seçilmiştir. $\mathrm{Bu}$ veri adları ve sayıları derivasyonlarla birlikte Tablo 1'de verilmiștir. 
European ST-T veritabanında V1, V2, V3, V4, V5 derivasyonları kullanılmıştır. Bu verilerden 111688 tane ST yükselmesi veya düşmesi olan R-R aralıkları seçilmiştir. $\mathrm{Bu}$ veri adları ve toplam sayıları derivasyonlarla birlikte Tablo 2'de verilmiştir. MIT$\mathrm{BIH}$ veritabanındaki sinyallerin örnekleme frekansı $360 \mathrm{~Hz}$ iken European ST-T veritabanındakilerin 250 Hz'dir.

Tablo 1. MIT-BIH Aritmi Veritabanından Alınan Kayıtlar

\begin{tabular}{lll}
\hline Derivasyon & Veri Adl & Toplam \\
\hline V1 & $101,105,106,107,108,109,111$, & 92157 \\
& $112,113,115,116,118,119,121$, \\
& $122,200,201,202,203,205,207$, \\
& $208,209,210,212,213,214,215$, \\
& $217,219,220,221,222,223,228$, \\
V2 & $230,231,232,233,234$ & 7939 \\
V4 & $102,103,104,117$ & 1604 \\
V5 & 124 & 9988 \\
\hline \multicolumn{3}{r}{} \\
\hline
\end{tabular}

Tablo 2. European ST-T Veritabanından Alınan Kayıtlar

\begin{tabular}{|c|c|c|}
\hline Derivasyon & Veri Adı & Toplam \\
\hline V1 & $\begin{array}{l}\text { e0403,e0614,e0704,e0801, } \\
\text { e0808,e1302,e1304 }\end{array}$ & 11496 \\
\hline V2 & $\begin{array}{l}\text { e0305,e0306,e0415,e0515, } \\
\text { e0603,e0604 }\end{array}$ & 8245 \\
\hline V3 & e0106,e0110,e0129,e0302, & 6004 \\
\hline V4 & $\begin{array}{l}\text { e0104,e0105,e0107, e0108, } \\
\text { e0111,e0112,e0113, e0114, } \\
\text { e0116,e0118,e0119, e0121, } \\
\text { e0122,e0124,e0125, e0126, } \\
\text { e0127,e0139,e0147, e0148, } \\
\text { e0154,e0159,e0161, e0162, } \\
\text { e0163,e0166,e0170,e0501, } \\
\text { e0607 }\end{array}$ & 38349 \\
\hline V5 & $\begin{array}{l}\text { e0115,e0202,e0204,e0205, } \\
\text { e0206,e0207,e0208,e0210, } \\
\text { e0211,e0212,e0213,e0303, } \\
\text { e0304,e0305,e0306,e0403, } \\
\text { e0404,e0405,e0410,e0411, } \\
\text { e0415,e0417,e0418,e0515, } \\
\text { e0602,e0603,e0605,e0607, } \\
\text { e0609,e0613,e0613,e0614, } \\
\text { e0808,e1302,e1304 }\end{array}$ & 47594 \\
\hline
\end{tabular}

MIT-BIH Aritmi veritabanındaki hastalıklı ve sağlıklı veriler bir sınıf, European ST-T veritabanındaki aritmi olan veriler farklı bir sınıf olacak șekilde toplamda 2 sınıf olușturulmuștur. Buradaki amaç ST değişimini (yükselmesi veya düşmesi) sağlıklı verilerden ve diğer hastalıklardan ayırt etmektir. $\mathrm{Bu}$ amaçla MIT-BIH Aritmi veritabanındaki V1, V2, V4 ve V5 derivasyonundaki hastalıklı ve sağlıklı veriler bir sınıf olarak seçilmiştir. MIT-BIH Aritmi veritabanında V3 derivasyonuna ait kayıtlar bulunmadığı için V3 derivasyonu seçilememiştir. Diğer sınıfsa European ST-T veritabanındaki V1, V2, V3, V4 ve V5 derivasyonlarındaki ST değişimi (düşmesi veya yükselmesi) olan kayıtlar alınmıştır. Çalışmanın en önemli amaçlarından biri de derivasyondan bağımsızlıktır. $\mathrm{Bu}$ nedenle European ST-T veritabanındaki V3 çıkarılmamıştır.

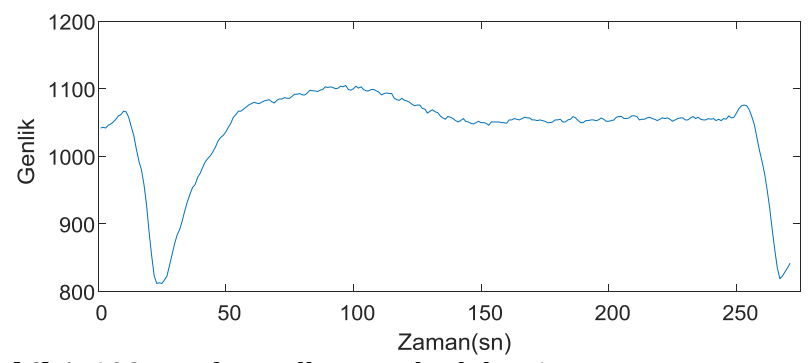

Şekil 1. 109m Left Bundle Branch Blok V1 Derivasyonu

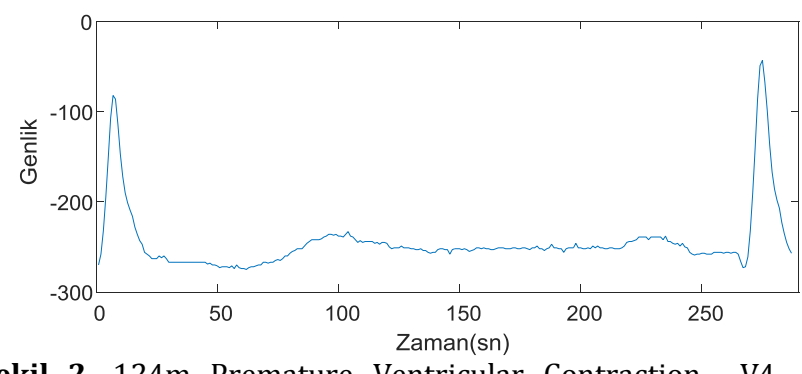

Sekil 2. $124 \mathrm{~m}$ Premature Ventricular Contraction V4 Derivasyonu

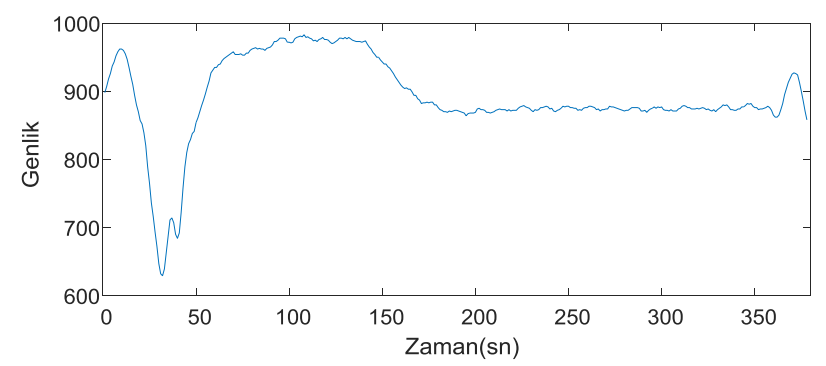

Şekil 3. e0104m ST Düşmesi V4 Derivasyonu

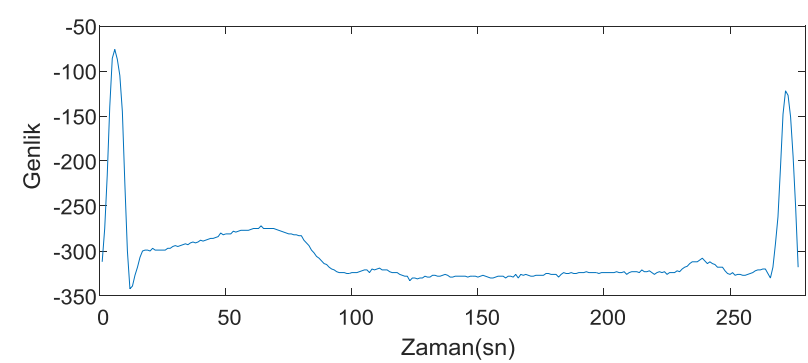

Şekil 4. e0104m ST Yükselmesi V4 Derivasyonu

Șekil 1,2,3 ve 4'te kullanılan veritabanlarından alınan örnek R-R aralıkları verilmiştir.

\section{2. Öznitelik çıkartımı}

EKG sinyallerinin genel șekli olmasına rağmen hastalıklı EKG sinyallerin şekilleri ve normal EKG sinyallerinin şekilleri birbirinden oldukça farklıdır. Aynı zamanda EKG sinyalleri alındığı ortama ve alındığı kişiye göre farklı şekillerde karşımıza çıkmaktadır.

EKG sinyalleri birçok gürültüden rahatlıkla etkilenmektedir. Bunlardan başlıcaları vücut ve elektrot arasındaki değişimlerden kaynaklı düșük frekans gürültüleri, hastanın hareketlerinden 
kaynaklı kas gürültüleri ve çevredeki cihaz veya şebekeden kaynaklı elektromanyetik gürültülerdir.

\subsubsection{Yumuşatılmış (smoothed) sahte (pseudo) wigner-ville zaman-frekans dağılımı}

EKG sinyalinin doğru bir şekilde analiz edilebilmesi yukarıdan bahsedilen sebeplerden dolayı oldukça zordur. $\mathrm{Bu}$ amaçla çalışmada geleneksel zaman frekans analizi yöntemlerinden farklı bir yöntem olan ve güçlü zaman-frekans çözünürlüğüne sahip Yumuşatılmış Sahte Wigner-Ville dönüşümü öznitelik çıkartımı aşamasında kullanılmıștır [24-25]. $X(\mathrm{t})$ sinyalinin Wigner-Ville dağılımı aşağıdaki formülde verilmiştir.

$$
\mathrm{WV}(\mathrm{t}, \omega)=\int_{-\infty}^{+\infty} s\left(\mathrm{t}+\frac{\tau}{2}\right) s^{*}\left(t-\frac{\tau}{2}\right) e^{-j \omega t} d \tau
$$

$s(\mathrm{t})$ : Sinyalin zaman serisi

$s^{*}(\mathrm{t})$ : Sinyalin karmaşı eşleniği

t: Anlık zaman

$\omega:$ Anllk frekans

$\tau$ : Zaman gecikmesi

Wigner-Ville dağılımının en önemli problemi çapraz terimledir. İki kuvvetli frekans bileşeni arasında oluşan çapraz terimler Yumuşatılmış Wigner-Ville dağılımı kullanılarak ortadan kaldırılabilir [26].

$$
\begin{gathered}
\operatorname{SPW}(\mathrm{t}, \omega)=\int_{-\infty}^{+\infty} \int_{-\infty}^{+\infty} s\left(\mathrm{t}+\frac{\tau}{2}\right) s^{*}(\mathrm{t}- \\
\left.\frac{\tau}{2}\right) g(v) h(\tau) e^{-j \omega t} d v d \tau
\end{gathered}
$$

$v$ : Dengeleme Frekansı

$g(v)$ : Zaman (Frekans uzayındaki yumuşatılmış pencere)

$h(\tau)$ : Frekans (Zaman uzayındaki yumuşatılmış pencere)

Pencereleme operasyonundaki $h$ ve $g$ yumuşatılmıș Wigner-Ville dağılımındaki zaman ve frekans uzayını ifade eder. Bu pencere seçme işlemiyle zaman frekans çözünürlüğü ve çapraz terim seviyeleri arasında istenilen denge sağlanmaya çalıșllır. Genelde dikdörtgensel veya Kaiser pencerelemeler tercih edilir [26]. Calıșmada Hamming pencereleme yöntemi kullanılmıştır.

R-R aralığı için sinyalin ortalama değeri hesaplanmıştır. Daha sonra standart sapmaya bölünerek şebeke veya cihazdan kaynaklı EKG sinyali genliğinde oluşabilecek yanlış kararların önüne geçilmesi sağlanmıştır. Bu işlem hem hızlı hem de sade olup gerçek-zamanlı uygulamalar için bir engel teşkil etmemektedir. Çalışmada güçlü zaman-frekans çözünürlüğüne sahip olan Yumuşatılmıș Sahte Wigner-Ville dönüşümü öznitelik çlkartımı aşamasında kullanılmıştır [24-25]. Her bir R-R aralığına Yumuşatılmış Sahte Wigner-Ville dönüşümü uygulanmiştır. Dönüşüm sonucunda 2 boyutlu katsayı matrisi elde edilmiştir. Bir sonraki aşamada çeşitli sebeplerden oluşan gürültünün etkisini azaltmak için band geçiren filtreleme uygulanmıştır. Frekans ekseninde ilk ve son $\% 3$ noktalarına tekabül eden frekanslar kesim frekansları olarak seçilmiștir. $\mathrm{R}-\mathrm{R}$ aralığının başlangıç ve bitiş noktaları çok büyük farklılıklar içermediği için başlangıç ve bitiş noktalarının \%3 kısımları ihmal edilmiștir. İșaretin geriye kalan kısmı 5 eşit aralığa bölünmüștür. Böylece dikey ekseni frekans değerleri, yatay ekseni ise zaman değerleri olan 5 bölge oluşturulmuştur. $\mathrm{Bu}$ bölgelerin öncelikle maksimum ve minimum katsayıları daha sonra ise türevinin maksimum ve minimum katsayıları belirlenmiştir. Daha sonra 10 tane maksimum 10 tane minimum değerler sırasıyla yazılarak her bir R-R aralığı için boyutu 20 olan öznitelik vektörü oluşturulmuştur.

\subsection{Sinıflandırma}

ST değişimi (düşmesi veya yükselmesi) V1, V2, V3, V4, V5 derivasyonlarında daha etkin gözükür. Bundan dolayl sinıfların biri sadece bu derivasyonlardaki ST değişimlerinden oluşturulmuştur. Diğer sınıf ise V1, V2, V4, V5 derivasyonlarına ait sağlıklı veri ve aritmilerden oluşturulmuştur.

Çalışmada önerilen yöntemin eğitim ve test performans analizini tespit etmek için Matlab yazılımındaki Classification Learner Toolbox'i kullanılmıștır. Eğitim așamasında 10-kat katlama (10-fold) yaklaşımı kullanılmıştır. Önerilen yöntemin sinıflandırma performans değerlendirmesi için Destek Vektör Makineleri (DVM), K-En Yakın Komşuluk (K-EYK) ve Topluluk Sinıflandırıcıları yöntemlerinden yararlanılmıștır.

Tüm DVM yöntemlerinde yumuşak-ayrım sabiti 1 olarak seçilmiştir. Doğrusal DVM yönteminde çekirdek fonksiyonu olarak (3)'deki formül kullanılmıştır. Buradaki K çekirdek fonksiyonunu ve $\mathrm{T}$ transpozeyi ifade eder.

$$
K\left(x_{1}, x_{2}\right)=x_{1}^{T} \cdot x_{2}
$$

Fine Gaussian (Radyal Tabanlı) ve Medium Gaussian (Radyal Tabanlı) DVM yöntemlerinde çekirdek fonksiyonu olarak (4)'deki formül kullanılmıştır. Bu formülde; $\gamma$ çekirdek ölçekleme değeridir. Fine Gaussian (Radyal Tabanlı) DVM yönteminde $\gamma_{\text {değeri }}$ $\frac{\sqrt{\alpha}}{4}$ eşitlenmiştir. $\alpha$ değeri eğitim verisindeki öznitelik vektörünün sütün sayısını ifade eder. Önerilen yöntemde $\alpha=20$ 'dir.

$$
K\left(x_{1}, x_{2}\right)=\exp \left(-\gamma\left\|x_{1}-x_{2}\right\|^{2}\right)
$$

Medium Gaussian (Radyal Tabanlı) DVM'de çekirdek ölçekleme değeri $\sqrt{\alpha}$ olarak seçilmiştir. Kubik ve Kuadratik DVM yönteminde çekirdek fonksiyonu olarak (5)'deki eșitlik kullanılmıștır. Kübik DVM 
yönteminde $\mathrm{p}=3$, Kuadratik DVM'de ise $\mathrm{p}=2$ olarak seçilmiştir.

$$
K\left(x_{1}, x_{2}\right)=\left(1+x_{1}^{T} x_{2}\right)^{p}
$$

K-EYK sınıflandırma yöntemlerinde farklı k değerleri ve farklı uzaklı hesaplama yöntemleri kullanılmamıştır. Fine K-EYK, Medium K-EYK, Coarse K-EYK algoritmalarında uzaklık hesaplamasında Öklid uzaklığı kullanılmıștır ve sırasıyla $\mathrm{k}=1, \mathrm{k}=10$ ve k=100 olarak seçilmiştir. Öklid uzaklığı (6)' daki formülde $\mathrm{p}=2$ seçilerek elde edilir.

$$
\left(\sum_{i=1}^{n}\left|x_{i}-y_{i}\right|^{p}\right)^{1 / p}
$$

Kosinüs K-EYK algoritmasında uzaklık hesaplamasında kosinüs uzaklığı kullanılmıștır. Kosinüs uzaklığı (7)'deki gibi ifade edilebilir burada T transpozeyi ifade eder ve $\mathrm{k}=10$ seçilmiştir.

$$
\left(1-\frac{X . Y^{T}}{\sqrt{\left(X . X^{T}\right)}\left(Y . Y^{T}\right)}\right)
$$

Kubik K-EYK algoritmasında uzaklık hesaplamasında (6)'daki Minkowski uzaklığı $\mathrm{p}=3$ ve $\mathrm{k}=10$ olarak seçilmiştir. Weighted K-EYK algoritmasında uzaklık hesaplamasında Öklid uzaklığı kullanılmıştır ve $\mathrm{k}=10$ seçilmiştir. Diğer K-EYK yöntemlerinden farklı olarak Komşuluk ağırlıklandırılması yapılmıș ve ağırlık değeri $\frac{1}{d^{2}}$ olarak seçilmiştir.

Sınıflandırıcı Toplulukları sınıflandırma işleminin tek bir sinıflandırma algoritmasıyla yapmak yerine; birçok sınıflandırma algoritması sonucunda elde edilen sonuçların birleştirilmesine yönelik yöntemlerdir [27].

Boosted Trees sınıflandırma yönteminde; AdaBoost algoritması Karar Ağacı sinıflandırma yöntemiyle kullanımıştır. AdaBoost algoritmasında eğitim veri setinden bir sinıflandırıcı oluşturulur bu sınıflandırıcının test sonucu başarısına göre, ilk iterasyonda yanlış sınıflandırılmış örnekler bir sonraki iterasyonda başarılı olsun diye ağırlıkları artırllır. Önerilen yöntemde Boosted Trees sınıflandırma performans değerlendirmesinde iterasyon sayısı 50 ve oluşturulacak ağaç sayısı 100 olarak alınmıştır [28].

Bagged Trees sınıflandırma yönteminde; Rastgele Ormanlar(RO) algoritması Karar Ağacı sınıflandırma yöntemiyle kullanılmıștır. RO, Bagging (Torbalama) yöntemine rastgelelik özelliği eklenerek geliștirilmiş şeklidir. $\mathrm{Bu}$ yöntemde ağacın her bir düğümünde rastgele olarak seçilen değişkenler arasında en iyi olanı kullanarak her bir dügümü dallara ayırır.
Rastgele özellik seçimi kullanılarak ağaçlar geliştirilir. $\mathrm{Bu}$ ağaçların geliştirilmesinde CART algoritması kullanılır. RO algortimasında oluşturulacak olan ağaç sayısı 100 olarak seçilmiştir [29].

AltUzay K-EYK yönteminde Rastgele Alt Uzay algoritması K-EYK sinıflandırma yöntemiyle kullanılmıștır. Rastgele Alt Uzay algoritmasında Ho tarafından önerilmiştir. $\mathrm{Bu}$ yöntemde eğitim kümesindeki tüm örnekler verilmektedir ancak bu örneklerin tüm özelliklerinin verilmesi yerine rastgele seçilen özellikleri alınmaktadır. Bu şekilde her seferinde sinıflandırma yöntemlerine farklı uzaylardaki verilerin dağılımlar verilip olaya farklı açllardan bakılabilmektedir [30]. Önerilen yöntemde AltUzay K-EYK siniflandırma performans değerlendirmesinde; K-EYK komşu sayısı 100 ve alt uzay boyutu gerçek uzay boyutun yarısı olan 10 olarak alınmıştır.

\section{Bulgular}

Önerilen yöntemi test etmek için birçok sınıflandırma algoritması kullanılmıştır. Sınıflandırma yöntemleri anlam karışıklılığına sebep olmamak için İngilizce isimleri ile verilmiştir.

Başlangıçta oluşturduğumuz 111688 aritmili ve sağlıklı veri, sınıf 1 olarak diğer 111688 ST değişimi olan veriler sınıf 2 olarak atanmıștır. Daha sonra bu veriler rastgele karıştırılarak 223376 değerine sahip öznitelik seti hazırlanmıștır. Eğitim doğruluğu ve test doğruluğu, duyarlılığı, özgüllüğü aşağıdaki formüllerden yararlanılarak hesaplanmıştır.

$$
\begin{aligned}
& \text { Doğruluk }=\frac{G P+G N}{G P+S P+S N+G N} \\
& \text { Duyarlllık }=\frac{G P}{G P+S N} \\
& \text { Özgüllük } k=\frac{G N}{G N+S P}
\end{aligned}
$$

GP: Gerçek pozitiflerin sayısı

SP: Sahte pozitiflerin sayısı

SN: Sahte negatiflerin sayısı

GN: Gerçek negatiflerin sayısı

Daha sonra 2 sınıftan da eşit sayıda veri alındığı için yaygınlı (prevalence) değeri 0.5 olarak bulunmuştur. Duyarlılık, özgüllük ve yaygınlık değerleri kullanılarak pozitif öngörü değerler de aşağıda ki formüllerden yararlanarak hesaplanmıștır. Bahsedilen işlem 3 defa tekrarlanarak değerlerin ortalama ve standart sapmaları Tablo 3 'de verilmiştir.

$$
\text { Pozitif Öngörü Değer }=\frac{D \times Y}{D \times Y+(1-\ddot{0}) \times(1-Y)}
$$

Duyarlılık:D

Yayginlık:Y

Özgüllük:Ö 


\section{Tartışma ve Sonuç}

Tablo 3'den de görüldüğü gibi doğruluk, duyarlılık, özgüllük ve pozitif öngörü değer sırasıyla \%93,80 ve $\% 98,78, \% 93,46$ ve $98,84 \%, \% 94,15$ ve $\% 99,58$, $\% 94,40$ ve \%99,56 arasında değişmektedir. En iyi sınıflandırma sonuçlarının, Destek Vektör Makineleri (DVM) algoritmalarındaki Kübik DVM yönteminde; K- En Yakın Komşuluk algoritmalarındaki Weighted (K-EYK) yönteminde; Sinıflandırıcı Toplulakları algoritmalarındaki Bagged Trees yönteminde olduğu görülmüștür. Bu algoritmaların doğruluk, duyarlılık, özgünlük değerlerinin standart sapmaları sırasıyla maksimum 0,17, 0,16, 0,17 değerlerinde olduğu gözlemlenmiştir. Standart sapmaların düşük olması önerilen yöntemin kararlı bir şekilde çalıştığını göstermektedir.

Tele-tıp uygulamalarının temel amaçlarından biri de kritik bir durumda hastaya bir an önce müdahale edip gerekli önlemleri almak olduğu için aritmi tespit hızı oldukça önemlidir. Bu çalışmada ST değişimin algılanabilmesi için 3 temel süre göz önünde bulundurulmuştur. Birincisi R-R tepeleri hesaplanırken geçen süre, ikincisi öznitelik çlkartılırken geçen süre ve sonuncusu da test için geçen süredir. $\mathrm{Bu}$ çalışmada $\mathrm{R}$ tepeleri veritabanından direk alınmıştır.

Tablo 3. Önerilen Yöntemin Performans Analizi

\begin{tabular}{|c|c|c|c|c|c|}
\hline \multirow[b]{2}{*}{ Sınıflandırma Yöntemi } & \multirow{2}{*}{$\begin{array}{c}\text { Eğitim } \\
\begin{array}{c}\text { Doğruluk } \\
(\%)\end{array}\end{array}$} & \multicolumn{4}{|c|}{ Test } \\
\hline & & $\begin{array}{c}\text { Doğruluk } \\
(\%)\end{array}$ & $\begin{array}{c}\text { Duyarlılık } \\
(\%)\end{array}$ & $\begin{array}{c}\text { Özgüllük } \\
(\%)\end{array}$ & $\begin{array}{l}\text { PPD } \\
(\%)\end{array}$ \\
\hline \multicolumn{6}{|c|}{ Destek Vektör Makineleri (DVM) } \\
\hline Kuadratik & $98,1 \pm 0,6$ & $97,69 \pm 0,02$ & $97,92 \pm 0,05$ & $97,43 \pm 0,04$ & 97,44 \\
\hline Kubik & $98,73 \pm 0,06$ & $98,76 \pm 0,04$ & $98,77 \pm 0,02$ & $98,83 \pm 0,07$ & 98,83 \\
\hline Fine Gaussian & $98,76 \pm 0,06$ & $97,78 \pm 0,04$ & $95,98 \pm 0,07$ & $99,58 \pm 0,01$ & 99,56 \\
\hline Medium Gaussian & $98,7 \pm 0,00$ & $98,72 \pm 0,02$ & $98,62 \pm 0,05$ & $98,82 \pm 0,02$ & 98,82 \\
\hline Doğrusal & $93,73 \pm 0,06$ & $93,80 \pm 0,02$ & $93,46 \pm 0,08$ & $94,15 \pm 0,13$ & 94,40 \\
\hline \multicolumn{6}{|c|}{ K-En Yakın Komșuluk (K-EYK) } \\
\hline Fine & $98,6 \pm 0,00$ & $98,65 \pm 0,00$ & $98,84 \pm 0,00$ & $98,47 \pm 0,00$ & 98,48 \\
\hline Medium & $98,63 \pm 0,06$ & $98,56 \pm 0,03$ & $98,65 \pm 0,02$ & $98,67 \pm 0,03$ & 98,67 \\
\hline Coarse & $97,97 \pm 0,21$ & $98,05 \pm 0,17$ & $98,24 \pm 0,16$ & $97,85 \pm 0,17$ & 97,86 \\
\hline Cosine & $98,5 \pm 0,00$ & $98,52 \pm 0,04$ & $98,53 \pm 0,05$ & $98,51 \pm 0,04$ & 98,51 \\
\hline Cubic & $98,46 \pm 0,06$ & $98,48 \pm 0,04$ & $98,40 \pm 0,08$ & $98,51 \pm 0,01$ & 98,51 \\
\hline Weighted & $98,7 \pm 0,00$ & $98,69 \pm 0,02$ & $98,73 \pm 0,01$ & $98,56 \pm 0,02$ & 98,56 \\
\hline \multicolumn{6}{|c|}{ Sınıflandırıcı Toplulukları } \\
\hline Bagged Trees & $98.73 \pm 0.06$ & $98.78 \pm 0.02$ & $98.55 \pm 0.06$ & $99.01 \pm 0.02$ & 99.01 \\
\hline Subspace k-NN & $98.63 \pm 0.06$ & $98.71 \pm 0.04$ & $98.58 \pm 0.04$ & $98.83 \pm 0.04$ & 98.83 \\
\hline Boosted Trees & $95.1 \pm 0.00$ & $95.18 \pm 0.00$ & $94.01 \pm 0.00$ & $96.35 \pm 0.00$ & 96.43 \\
\hline
\end{tabular}

*PPD: Pozitif Öngörü Değer

Tablo 4. Önerilen Yöntemin Diğer Çalışmalarla Karşılaștırılması

\begin{tabular}{|c|c|c|c|c|c|}
\hline Yazar & Veritabanı & Doğruluk (\%) & Duyarlılık (\%) & Özgüllük (\%) & PPD (\%) \\
\hline [3] & $\begin{array}{c}\text { European ST-T 17,314 EKG } \\
\text { atımı }\end{array}$ & 90,1 & - & - & - \\
\hline [6] & $\begin{array}{c}\text { Physikalisch-Technische } \\
\text { Bundesanstalt Veritabanı } \\
148 \text { aritmi ve } 52 \text { sağlıklı veri }\end{array}$ & 94,4 & - & - & - \\
\hline [17] & $\begin{array}{c}\text { European ST-T veritabanı } 10 \\
\text { veri normal veya aritmi } \\
86384 \text { kalp atımı }\end{array}$ & - & 87 & 93 & - \\
\hline [18] & Long Term ST-T Veritabanı & - & 83,33 & - & 77,31 \\
\hline [19] & $\begin{array}{l}\text { Long Term ST-T Veritabanı } \\
\text { European ST-T veritabanı } 14\end{array}$ & - & 89 & - & 85 \\
\hline$[20]$ & $\begin{array}{l}\text { veri aritmi MIT-BIH } \\
\text { veritabanı } 24 \text { veri normal }\end{array}$ & - & 100 & - & 93,33 \\
\hline [21] & European ST-T veritabanı & - & 93,8 & - & 78,5 \\
\hline [22] & $\begin{array}{c}\text { European ST-T veritabanı } 10 \\
\text { veri43,876 ST segmenti }\end{array}$ & - & 98,12 & 98,16 & - \\
\hline $\begin{array}{l}\text { Önerilen } \\
\text { Yöntem }\end{array}$ & $\begin{array}{c}\text { European ST-T veritabanı } \\
\text { MIT-BIH veritabanı }\end{array}$ & 98,78 & 98,55 & 99,01 & 99,01 \\
\hline
\end{tabular}


Öznitelik çıkartılırken 2 tane veritabanından yararlanılmıștır. MIT -BIH veritabanında bir RR aralığı için öznitelik çıkartımı için gerekli süre 2.9 saniye European ST-T veritabanı içinse 1.6 saniyedir. Test süresi oldukça kısa olduğu için ihmal edilebilir. Toplam süre yaklaşık olarak 3 atım için geçen süre kadardır. Hem bu süreye bakıldığında hem de yöntemin doğrudan bir ön işlemeye gerek duymadığı göz önüne alındığında Tele-tıp uygulamaları için uygun olduğu görülmektedir.

Çalışmada öznitelik çıkartımı süreleri hesaplanırken kullanılan bilgisayarın ișlemcisi 64 bit Intel(R) Core, 998 MHz. RAM'i ise 4,00 GB' dır. Yöntemde kullanılan algoritma Matlab ortamında optimize edilmeyen kodlarla geliştirildiği için hesaplama süresi istenilen değerde çıkmamıştır. $\mathrm{Bu}$ süre $\mathrm{C}$ programla dili kullanılarak ve Wigner-Ville katsayılarının sayısı azaltılarak düşürülebilir.

Sınıflandırmada kullanılan veriler bay-bayan farklı cinsiyetlere sahip, farklı yaş gruplarındaki hastalardan alınmıştır. Verilerin farklı hastalardan olmasına rağmen yüksek bir performans sağlanmıştır. Tablo 4'de gösterilen literatürdeki diğer çalışmalarla kıyaslandığında önerilen yöntemin doğruluk, duyarlılık, özgüllük ve pozitif öngörü değer açısından başarısının daha yüksek olduğu görülmektedir. Ayrıca önerilen yöntemde diğer yöntemlere göre daha büyük veritabanı kullanılmıştır. Tablo 4'te görüldüğü gibi sadece bir çalışmanın [20] doğruluk değerinin önerilen yöntemden daha iyi sonuç verdiği görülmüştür. Ancak bu çalışmanın pozitif öngörü değerinin önerilen yönteme göre oldukça düşük olduğu görülmüştür ve çalışmanın gerçek zamanlı olup olmadığı hakkında bir bilgiye yer verilmemiştir.

\section{Teşekkür}

$\mathrm{Bu}$ çalışma 1003 programı kapsamındaki 114E452 nolu projeyle TÜBİTAK tarafından desteklenmiştir.

\section{Kaynakça}

[1] Centers for Disease Control and Prevention CDC. 2003. Trends in aging--United States and worldwide. MMWR. Morbidity and mortality weekly report, 52(6), 101.

[2] WHO. 2016. Cardiovascular diseasen http://www.who.int/cardiovascular_diseases/e n (Erişim Tarihi: 27.12.2016).

[3] Xu, M., Wei, S., Qin, X., Zhang, Y., Liu, C. 2015. Rule-Based Method for Morphological Classification of ST Segment in ECG Signals. Journal of Medical and Biological Engineering, 35(6), 816-823.

[4] Thygesen, K., Alpert, J. S., White, H. D. 2007. Universal definition of myocardial infarction. Journal of the American College of Cardiology, 50(22), 2173-2195.
[5] Roger, V. L., Go, A. S., Lloyd-Jones, D. M., Adams, R. J., Berry, J. D., Brown, T. M., Fox, C. S. 2011. Heart disease and stroke statistics-2011 update a report from the American Heart Association. Circulation, 123(4), e18-e209.

[6] Liu, B., Liu, J., Wang, G., Huang, K., Li, F., Zheng, Y., Zhou, F. 2015. A novel electrocardiogram parameterization algorithm and its application in myocardial infarction detection. Computers in biology and medicine, 61, 178-184.

[7] Wimmer, N. J., Scirica, B. M., Stone, P. H. 2013. The clinical significance of continuous ECG (ambulatory ECG or Holter) monitoring of the ST-segment to evaluate ischemia: a review. Progress in cardiovascular diseases, 56(2), 195-202.

[8] Wootton, R. 2012. Twenty years of telemedicine in chronic disease management-an evidence synthesis. Journal of telemedicine and telecare, 18(4), 211-220.

[9] Rabbani, H., Mahjoob, M. P., Farahabadi, E., Farahabadi, A., Dehnavi, A. M. 2011. Ischemia detection by electrocardiogram in wavelet domain using entropy measure. Journal of Research in Medical Sciences, 16(11).

[10] Ranjith, P., Baby, P. C., Joseph, P. 2003. ECG analysis using wavelet transform: application to myocardial ischemia detection. ITBMRBM, 24(1), 44-47.

[11] Afsar, F. A., Arif, M., Yang, J. 2008. Detection of ST segment deviation episodes in ECG using KLT with an ensemble neural classifier. Physiological measurement, 29(7), 747.

[12] Smrdel, A., Jager, F. 2004. Automated detection of transient ST-segment episodes in $24 \mathrm{~h}$ electrocardiograms. Medical and Biological Engineering and Computing, 42(3), 303-311.

[13] Goletsis, Y., Papaloukas, C., Fotiadis, D. I., Likas, A., Michalis, L. K. 2004. Automated ischemic beat classification using genetic algorithms and multicriteria decision analysis. IEEE transactions on Biomedical Engineering, 51(10), 1717-1725.

[14] Andreao, R. V., Dorizzi, B., Boudy, J., Mota, J. C. M. 2004. ST-segment analysis using hidden Markov Model beat segmentation: application to ischemia detection. In Computers in Cardiology, 2004 (pp. 381-384). IEEE.

[15] Correa, R., Arini, P. D., Correa, L. S., Valentinuzzi, M., Laciar, E. 2014. Novel technique for ST-T interval characterization in patients with acute myocardial ischemia. Computers in biology and medicine, 50, 49-55.

[16] Chang, P. C., Lin, J. J., Hsieh, J. C., Weng, J. 2012. Myocardial infarction classification with multilead ECG using hidden Markov models and Gaussian mixture models. Applied Soft Computing, 12(10), 3165-3175. 
[17] Exarchos, T. P., Papaloukas, C., Fotiadis, D. I., Michalis, L. K. 2006. An association rule miningbased methodology for automated detection of ischemic ECG beats. IEEE Transactions on Biomedical Engineering, 53(8), 1531-1540.

[18] Dranca, L., Goni, A., Illarramendi, A. 2009. Realtime detection of transient cardiac ischemic episodes from ECG signals. Physiological measurement, 30(9), 983.

[19] Tang, X., Xia, L., Liu, W., Peng, Y., Gao, T., Zeng, Y. 2012. An approach to determine myocardial ischemia by hidden Markov models. Computer methods in biomechanics and biomedical engineering, 15(10), 1065-1070.

[20] Al-Fahoum, A., Al-Fraihat, A., Al-Araida, A. 2014. Detection of cardiac ischaemia using bispectral analysis approach. Journal of medical engineering \& technology, 38(6), 311-316.

[21] Papaloukas, C., Fotiadis, D. I., Liavas, A. P., Likas, A., Michalis, L. K. 2001. A knowledge-based technique for automated detection of ischaemic episodes in long duration electrocardiograms. Medical and Biological Engineering and Computing, 39(1), 105-112.

[22] Kumar, A., Singh, M. 2016. Ischemia detection using Isoelectric Energy Func-tion. Computers in biology and medicine, 68, 76-83.

[23] Physionet. 2016. ECG Database. http://physionet.org/physiobank/database/\#ec g (Erişimim Tarihi: 27.12.2016).

[24] Kayıkçığlu, İ., Akdeniz, F., Kayıkçıŏlu, T. 2016. Wigner-Ville distribution based ECG arrhythmia detection for telemedicine applications. In Signal Processing and Communication Application Conference (SIU), 2016 24th (pp. 2045-2048). IEEE.

[25] Akdeniz, F., Kayıkçıŏ̆lu, İ., Kaya, İ., Kayıkçıoğlu, T. 2016. Using Wigner-Ville distribution in ECG arrhythmia detection for telemedicine applications. In Telecommunications and Signal Processing (TSP), 2016 39th International Conference on (pp. 409-412). IEEE.

[26] Cohen, L. 1995. Time-Frequency Analysis: Theory and Applications, Prentice-Hall, Inc.

[27] Brown, G. 2011. Ensemble learning, Encyclopedia of Machine Learning, Springer US,(2011) 312-320.

[28] Rokach, L. 2010. Ensemble-based classifiers, Artificial Intelligence Review, 33,1 (2010)1-39.

[29] Breiman, L. 2001. Random forests, Machine learning, 45,1 (2001) 5-32.

[30] Ho, T. K. 1998. The random subspace method for constructing decision forests, IEEE Transactions on Pattern Analysis and Machine Intelligence, 20,8 (1998) 832-844. 